\title{
Gamma-ray burst afterglow observations using AXAF and ACIS
}

\author{
G.P. Garmire \\ Pennsylvania State University, 525 Davey Lab, University Park, PA 16802, U.S.A. \\ e-mail: garmire@astro.psu.edu
}

Received December 18, 1998; accepted March 10, 1999

\begin{abstract}
With the successful launch of AXAF next summer, it will be possible to observe the X-ray afterglow from gamma-ray bursts with much improved positional accuracy $(\sim 1$ arcsec $)$ and sensitivity $(2-10 \mathrm{keV}$, $1.510^{-14} \mathrm{ergs} / \mathrm{cm}^{2} / \mathrm{s}$ in $\left.10 \mathrm{ksec}\right)$. A typical burst afterglow should be observable within about 10 hours after the position has been determined by BeppoSAX or any other satellite capable of determining the position to within about 5 arcmin and provide about 1000 counts in a $10 \mathrm{ksec}$ integration using the ACIS on AXAF. To constrain the decrease of intensity with time, using existing data from BeppoSAX and ASCA, the burst should produce a measurable flux for up to about 4 months after the gamma-ray event for an observing time of about $40 \mathrm{ksec}$, if a simple extrapolation of the observed fall-off with time is assumed. Depending upon a number of assumptions, there is a slight possibility of observing iron lines from the gas immediately surrounding the gamma-ray burst source using a long exposure (>100 ksec).
\end{abstract}

Key words: gamma-ray bursts

\section{Introduction}

The Italian-Dutch satellite, Beppo-SAX, transformed the field of gamma-ray burst studies by discovering the $\mathrm{X}$-ray afterglow from the gamma-ray burst and determining the position of the burst to arcminute accuracy. The ensuing optical observations then showed conclusively that the bursts originated at large redshifts, and were of extreme luminosity. The predictions by Paczyński \& Rhoads (1993); Katz (1994); Mészáros \& Rees (1998); and Vietri (1997); have largely been born out by observations. The general decrease in the X-ray intensity has been found to follow a powerlaw of the form $t^{-\alpha}$ where the value of $\alpha$ ranges between 1.1 and 1.9 for the few bursts that have been observed (Costa et al. 1997; Costa et al. 1998; Yoshida et al. 1998). So far the bursts in X-rays have been followed for about 10 days before they fade below the current instrument sensitivities. By following the burst for several months using AXAF, the powerlaw decay can be tested to greater limits. The great advantage of AXAF is the ability to position the gamma-ray burst to arcsecond accuracy within a thousand seconds. This makes the association with a galaxy or any other object virtually a hundred percent clear, since the mean spacing between the faintest galaxies is of the order of several arcseconds. Since AXAF is so sensitive, it is feasible to wait until an optical identification has been made or not. If no optical identification has been made, then AXAF can position the gamma-ray burst and still determine if it is associated with a galaxy. There could be a class of gamma-ray bursts that fade very rapidly in the optical band or are intrinsically fainter because of the surrounding medium (Panaitescu et al. 1998).

\section{Observational strategy}

After a gamma-ray burst position has been determined by BeppoSAX or RXTE to an accuracy of about five arcminutes, AXAF can be pointed to the position within less than a day and perhaps within eight hours. Even for the most pessimistic predictions of Panaitescu et al. (1998), a 10 ksec exposure should easily detect the burst (see Fig. 1).

For typical X-ray afterglow intensities observed within one day after the gamma-ray event, about $10 \mathrm{ksec}$ of observing time should collect about $10^{3}$ counts for spectral analysis. This should be enough to determine spectral slope and absorption cut-off for the burst.

Spectral features from the surrounding medium of the burst are very difficult to predict, since the environment of the preburst object is unknown. Mészáros \& Rees (1998) have given some estimates of iron line emission from a variety of environments. They estimate that the flux in the line would be of the order of $310^{-16} \mathrm{ergs} / \mathrm{cm}^{2} / \mathrm{s}$ for a unit density of the surrounding medium. If the medium were more dense, as in an HII region, then one could get more than an order of magnitude increase in flux. 
The X-ray Decay of Gamma-Ray Burst Afterglows Data from the 970228,970508 , and 970828 Bursts

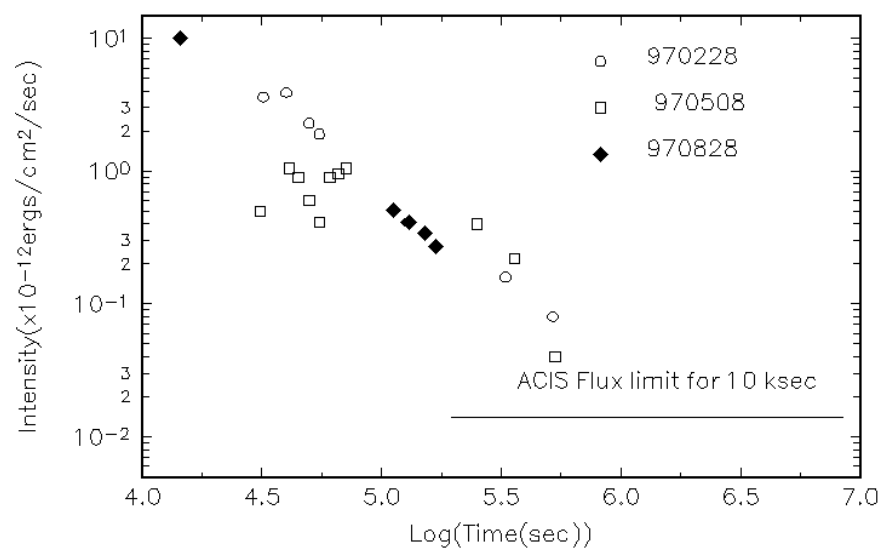

Fig. 1. The sensitivity of ACIS for detecting GRB afterglows in $10^{4} \mathrm{~s}$. This limit scales as $t^{-1}$ up to $10^{5} \mathrm{~s}$

The process that creates the iron lines needs to be examined in some detail. The gamma-ray burst is so intense within about a parsec of the source that it creates what in laser physics is called "self-induced transparency". In the case of the gamma-ray burst the photon flux is so intense that all of the atoms are stripped of their electrons before the burst photons completely pass a given point in space. Liang \& Kargatis (1994) noted this effect. The other point is that the cross section of the atomic electrons decreases rapidly with increasing energy resulting in the removal of the electrons from the outside of the atom toward the inside which reduces the intensity of fluorescent $\mathrm{K}$ photons by about a factor of twenty. The electrons from the atoms are ejected as photoelectrons or Compton recoil electrons and are not available to emit fluorescent photons. By formulating the fluorescent flux in terms of the time after the burst, it can be shown that the flux scales as $t^{2}$ for the time after the burst, so that the chance of detecting any fluorescent photons increases with time up to the point where the burst wave escapes from the local medium or the photon flux in the burst falls below the point where it ionizes all of the atoms in the surrounding medium. The decrease in absorption of the burst spectrum with time through the burst is a good indication that self-induced transparency is playing a role in the burst spectral evolution. To observe the fluorescent photons with the greatest probability, it is best to choose a burst that appears to be within a galaxy, that shows strong spectral evolution, and then wait for a number of months to make the observation. Even so, it will require a very long exposure, $\sim 10^{5} \mathrm{~s}$.

\section{Conclusions}

The observation of a gamma-ray burst afterglow using AXAF can be used for several purposes. The use of the time depends upon how long the observation time is. For a very short observation $\sim 10^{3} \mathrm{~s}$, obtaining the burst position shortly after the burst is the best use of the time. If the optical counterpart has already been detected, then AXAF can add little to the observation. If no optical counterpart is detected within a day, then the accurate X-ray position will permit association to be made with a galaxy to a very deep limiting magnitude, $28-30 m_{v}$. For the soft gamma-ray repeaters which are observable out to $\sim 1 \mathrm{Mpc}$ a significant flux of fluorescent photons might be observable for a time of $\sim$ days.

Acknowledgements. Part of this work was supported by NASA under contract No. NAS8-38252. The author wishes to thank Audrey Garmire for some useful calculations and comments, and Neil Gehrels and Fiona Harrison for a useful discussion.

\section{References}

Costa, et al., 1997, Nat 387, 783-785

Costa, et al., 1998, Gamma-ray bursts; $4^{\text {th }}$ Huntsville Symposium, Meegan C.A., et al. (eds.) AIP

Liang E.P., Kargatis V., 1994, ApJ 432, L111-L114

Katz J.I., 1994, ApJ 432, L107-L109

Mészáros P., Rees M., 1998, MNRAS 299, L10-L14

Paczyński B., Rhoads J.E., 1993, ApJ 418, L5-L8

Panaitescu A., Mészáros P., Rees M., 1998, ApJ 503, 314-324

Vietri M., 1997, ApJ 488, L105-L108

Yoshida A., et al., 1998, Gamma-ray bursts; $4^{\text {th }}$ Huntsville Symposium, Meegan C.A., et al. (eds.) AIP 